$50 \%$ of hypothermic piglets c.f. $83 \%$ in normothermic piglets. At $48 \mathrm{~h}$ post insult both groups showed a maximum of epileptic activity. The neurobehavioral score in the normothermic piglets showed an earlier return to baseline compared to the hypothermic piglets.

Conclusions Electrographic seizure burden was decreased following TH. aEEG background pattern and neurobehaviour score recovered earlier in normothermic piglets, suggesting that in the clinical situation conclusions based on aEEG and neurological examination should not be performed too early.

\section{HIGH DOSE INTRAVENOUS MELATONIN FOR AUGMENTATION OF HYPOTHERMIC NEUROPROTECTION LEADS TO HYPOTENSION}

doi:10.1136/archdischild-2012-302724.0303

M Ezzati, S Faulkner, K Broad, NJ Robertson. Institute for Women's Health, University College London, London, UK

Background Therapeutic hypothermia provides neuroprotection in infants with moderate to severe neonatal encephalopathy, however further treatments are necessary as hypothermic neuroprotection is not absolute.

Aim To assess the effect on mean arterial blood pressure of high dose intravenous melatonin $(20,10,5 \mathrm{mg} / \mathrm{kg})$ administered $10 \mathrm{~min}$ utes after hypoxia-ischemia.

Methods Male piglets underwent a hypoxic-ischemic insult and were then commenced on different doses of intravenous melatonin. Physiological measures such as mean arterial blood pressure and heart rate were measured. Hypothermia (core temperature $33.5^{\circ} \mathrm{C}$ ) was induced at 2-26h after hypoxia-ischemia.

Results $20 \mathrm{mg} / \mathrm{kg}$ melatonin induced a rapid reduction in blood pressure beginning at 1 hour following its administration (1-3h post administration; mean arterial blood pressure was reduced from 60 to $24 \mathrm{~mm} \mathrm{Hg}$. $10 \mathrm{mg} / \mathrm{kg}$ induced a gradual reduction in blood pressure at 1 hour post administration (1-4h post administration; mean arterial blood pressure was reduced from 55 to $31 \mathrm{~mm} \mathrm{Hg}$ ). However treatment with either 0 or $5 \mathrm{mg} / \mathrm{kg}$ melatonin had no effect on mean arterial blood pressure.

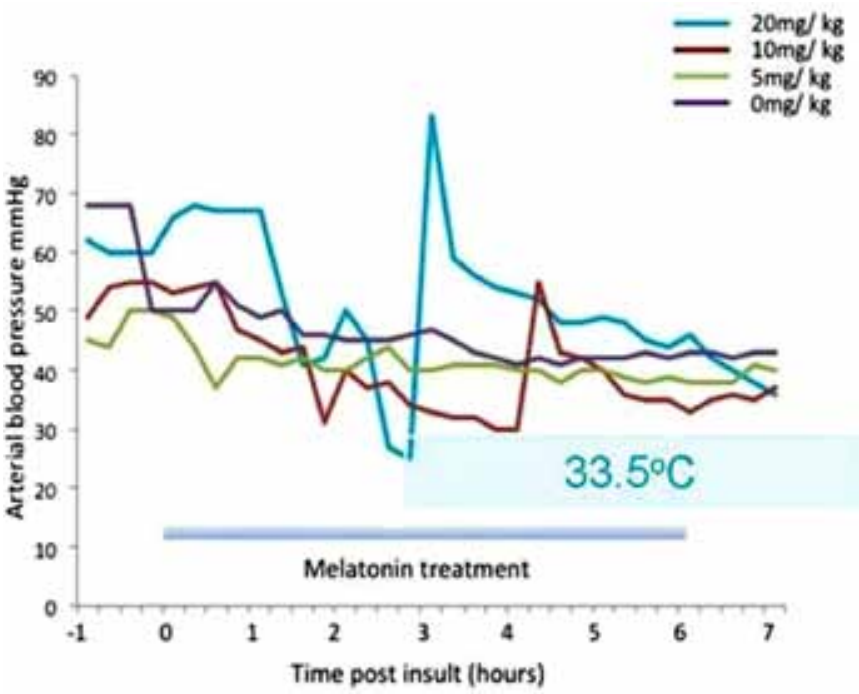

Abstract 303 Figure 1 Effect of different Melatonin doses on mean BP

Conclusion Intravenous doses of melatonin $(>5 \mathrm{mg} / \mathrm{kg}$ ) lead to hypotension following a hypoxic-ischemic insult when combined with hypothermia. Future pre-clinical studies of augmented hypothermic neuroprotection should be conducted using melatonin doses of $5 \mathrm{mg} / \mathrm{kg}$ or less.

\section{MINOCYCLINE ATTENUATES INJURY OF OLIGODENDROGLIAL PRECURSOR CELLS CAUSED BY OXYGEN-GLUCOSE-DEPRIVATION}

doi:10.1136/archdischild-2012-302724.0304

'T Schmitz, 'S Endesfelder, 'I Zaak, ${ }^{2}$ LJ Chew, ${ }^{1} \mathrm{C}$ Bührer. 'Department of Neonatology, Charité Universitätsmedizin Berlin, Berlin, Germany; ${ }^{2}$ Center for Neuroscience Research, Children's National Medical Center, Washington, DC, USA

Background Oxygen-glucose deprivation (OGD) is a widely used in vitro model for ischemic brain injury, which leads to cell death. Prevention and attenuation of brain injury by the tetracycline-antibiotic minocycline has been largely attributed to suppression of microglial activation.

Methods Using mono-cultures of rat oligodendroglial precursor cells (OPC) exposed to oxygen-glucose deprivation (OGD), we investigated direct effects of minocycline on survival, proliferation, and maturation of oligodendroglial lineage cells.

Results OGD for $2 \mathrm{~h}$ decreased the number of A2B $5+$ cells and the amount of proliferating Ki67+ A2B $5+$ cells by $50 \%$ which was both attenuated by minocycline in a dose-dependent fashion. The reduced numbers of $\mathrm{O} 4+$ cells at $72 \mathrm{~h}$ and of $\mathrm{O} 1+$ cells at $120 \mathrm{~h}$ after OGD were partially restored by minocycline. In OPC, OGD caused an increase of reactive oxygen species (ROS) production and of TUNEL-positive cell numbers which was abolished by minocycline, possibly via induction of superoxide dismutase. Minocycline also prevented OGD-induced downregulation of the expression of the transcription factors Sox10 and Olig2, and of the maturation markers 2'3' cyclic nucleotide phosphodiesterase (CNP) and myelin basic protein (MBP)

Conclusion The results demonstate that minocycline exerts direct protective actions on oligodendroglial lineage cells.

\section{DOPAMINE REDUCES WHITE MATTER INJURY IN HYPOXIC- ISCHAEMIA IN THE PRETERM LAMB}

doi:10.1136/archdischild-2012-302724.0305

F Wong, K Cassimally, A Azhan, T Samarasinghe, I Nitsos, A Walker, D Walker. The Ritchie Centre, Monash University, Melbourne, VIC, Australia

Background Dopamine is frequently used as inotropic agent in preterm infants. Its cardiovascular actions, as well as effects on neurovascular interactions may be neuroprotective during hypoxicischaemic events. Using a preterm lamb model we aimed to test the impact of intravenous dopamine on hypoxic-ischaemic brain injury. Method Nine fetal lambs (91-93d gestation) were instrumented with catheters in carotid artery and jugular vein, and an umbilical cord occluder. Four days after surgery, intravenous dopamine (DA, $10 \mu \mathrm{g} / \mathrm{kg} / \mathrm{min}, \mathrm{n}=5$ ) (or saline, $\mathrm{n}=4$ ) was commenced. then a hypoxic-ischaemic insult was induced with umbilical cord occlusion for 25 mins. Infusions were continued for another 72 hours before euthanasia. Fetal brains were collected for immunohistopathology. Results Dopamine infusion increased fetal heart rate $(184 \pm 1$ to $203 \pm 1 \mathrm{bpm}, \mathrm{p}<0.05)$ while arterial pressure was unchanged. Three animals in the DA group showed tachycardic response to cord occlusion, while the other two animals showed bradycardic response similar to the saline group. In the periventricular white matter, the saline group had higher number of microglia (lectin positive) than the DA group ( $10 \pm 3$ vs $6 \pm 2$ per $0.04 \mathrm{~mm}^{2}, \mathrm{p}<0.05$ ) The saline group tended to have shorter myelinated fibre lengths (CNPase) compared with the DA group $(15.0 \pm 2.0$ vs $18.4 \pm 5.7 \mu \mathrm{m}$ respectively, $\mathrm{p}=\mathrm{ns}$ ). No histological differences were evident between DA animals exhibiting a tachycardic or bradycardic response during cord occlusion.

Conclusions Intravenous dopamine reduces hypoxic-ischaemic white matter injury in preterm lambs, independent of the cardiovascular response during the hypoxic-ischaemia. 